\title{
Side-Chain Crystallization of Comb-Like Copolymers Obtained from Isopropenyltriazines with Comonomers Having Octadecyl Group
}

\author{
Hideo Kunisada, Yasuo Yuki, Shuji Kondo, \\ and Katsuya WADA \\ Department of Materials Science and Engineering, \\ Nagoya Institute of Technology, \\ Gokiso-cho, Showa-ku, Nagoya 466, Japan
}

(Received March 29, 1991)

\begin{abstract}
Copolymerizations of 2-amino-4-anilino-6-isopropenyl-1,3,5-triazine and 2amino-4-( $N$-octadecylanilino)-6-isopropenyl-1,3,5-triazine with octadecyl acrylate, octadecyl methacrylate, $\mathrm{N}$-octadecylacrylamide, and $\mathrm{N}$-octadecylmethacrylamide were carried out in order to investigate the side-chain crystallization of the resulting comb-like copolymers. Crystallization exothermic and melting endothermic peaks due to the side-chains of the comb-like copolymers were observed in differential scanning calorimetry (DSC) thermograms. When the content of isopropenyltriazine unit in the copolymer was high, glass transition temperature was observed above the melting point of the side-chain crystallites. The influence of monomer structure and copolymer composition on the crystallinity of the side-chains and glass transition temperature are discussed.

KEY WORDS Isopropenyltriazines / Long Alkyl Group / Comb-Like Copolymer / Side-Chain Crystallization / Glass Transition Temperature /
\end{abstract}

It is well known that long alkyl side-chains in comb-like polymer can be crystallized even if the main-chain is amorphous. This behavior would result in the dramatic change of mechanical property and permeability control of the polymers. However, it has been reported that glass transition of the conventional comb-like polymers such as poly(octadecyl acrylate), poly(octadecyl methacrylate), and poly $(N$-octadecylacrylamide $)$ is observed at the melting point of side-chains. ${ }^{1-4}$ Therefore, in order to apply the crystallization phenomenon to functional material, it is required that the glass transition temperature $\left(T_{\mathrm{g}}\right)$ of main-chain be higher than the melting point of the side-chains.

We studied the polymerization of isopropenyl-1,3,5-triazines and found that these polymers had the highest $T_{\mathrm{g}}$ and best thermal stability among vinyl polymers so-far studied owing to the rigidity and the hydrogen bonding of amino group. ${ }^{5-7}$ In our previous papers, we reported that side-chains of the comb-like polymers prepared from the homopolymerization $^{8}$ and copolymerization ${ }^{9}$ of 2-amino-4- $(N$ alkylanilino)-6-isopropenyl-1,3,5-triazines conaining a long alkyl group could be crystallized in a similar manner to conventional comb-like polymers in spite of high $T_{\mathrm{g}}$ of the main-chain. However, the crystallinity and melting point of the side-chains were lower than that of conventional comb-like polymers. Relatively flexible main-chain would be necessary to increase the crystallinity and melting point. The copolymers consisting of rigid monomer unit and flexible monomer unit seems to be effective for this purpose.

In order to prepare the comb-like copolymer having high $T_{\mathrm{g}}$ of main-chain and high melting point and crystallinity of side-chains, 
copolymerizations of isopropenyltriazines with monomers having octadecyl group such as octadecyl acrylate (OA), octadecyl methacrylate (OMA), $N$-octadecylacrylamide (OAAm), and $N$-octadecylmethacrylamide (OMAm) were carried out and side-chain crystallization of the resulting comb-like copolymers was studied.

\section{EXPERIMENTAL}

\section{Monomers}

2-Amino-4-anilino-6-isopropenyl-1,3,5-triazine $(\mathrm{AAIT})^{5}$ and 2 -amino-4-( $N$-octadecylanilino)-6-isopropenyl-1,3,5-triazine $(\mathrm{N} 18)^{8}$ were prepared as described in previous papers. OA and OMA were commercially available and purified by recrystallization from ethanol. OAAm and OMAm were prepared from octadecylamine with acryloyl chloride and metacryloyl chloride, respectively, and purified by recrystallization from acetone. ${ }^{10}$ The structure and abbreviation of the monomers used in this study are shown in Figure 1.

\section{Polymerization}

Homopolymerization and copolymerization were carried out using azobisisobutyronitrile (AIBN) as an initiator in $N, N$-dimethylacetamide (DMAc) or benzene at $60^{\circ} \mathrm{C}$. The monomer and AIBN concentrations were $0.4 \mathrm{moll}^{-1}$ and $1.4 \mathrm{mmoll}^{-1}$, respectively. The required volumes of monomer and AIBN solutions were charged into polymerization tubes and degassed by three alternate freezepump-thaw cycles. After being sealed, the tubes were placed in a constant temperature bath for specified times. The polymers were isolated by precipitating into ethanol and purified by reprecipitation. The composition of the copolymer was calculated by elemental analysis of carbon and nitrogen contents.

\section{Measurements}

Differential scanning calorimetry (DSC) measurements were performed on a Rigaku-
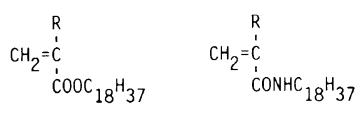

$O A(R=H)$

OMA $\left(\mathrm{R}=\mathrm{CH}_{3}\right)$

$$
\text { OAAm }(\mathrm{R}=\mathrm{H})
$$$$
\text { OMAm }\left(\mathrm{R}=\mathrm{CH}_{3}\right)
$$

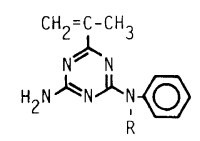

AAIT $(R=H)$

N18 $\left(R=n-C_{18} H_{37}\right)$
Figure 1. Structures and abbreviations of monomers.

Denki DSC-8230 at heating and cooling rates of $10^{\circ} \mathrm{C} \mathrm{min}{ }^{-1}$. Indium and benzoic acid were used as standards for calibration of temperature and heat energy. Molecular weights of the polymers were determined by gel permeation chromatography (GPC) on a TOSOH HLC803D with G2000H, G3000H, and GMH TSK gel columns and a differential refractometric detector in THF. The columns were calibrated with monodisperse polystyrene standards.

\section{RESULTS AND DISCUSSION}

Table I shows the results of homopolymerizations for OA, OMA, OAAm, OMAm, AAIT, and N18. Colorless solid polymers were obtained for all cases. The crystallization behavior of alkyl side-chains for the resulting comb-like polymers was investigated by DSC measurement. DSC curves of the comb-like polymers are shown in Figure 2 . The polymer sample was first heated from $-100^{\circ} \mathrm{C}$ to $+120^{\circ} \mathrm{C}$, then cooled to $-100^{\circ} \mathrm{C}$, and again heated to $+150^{\circ} \mathrm{C}$. In the DSC curve of poly(OA), endothermic peaks based on the melting of crystallized octadecyl side-chains were observed in the heating processes, and an exothermic peak based on the crystallization of the side-chains was observed during a cooling. Because of the same history of thermal treatment in DSC measurement, the second heating run was used for evaluation and comparison of the thermal properties of the comb-like polymers. The second heating thermograms of poly(OMA), poly(OAAm), poly(OMAm), and poly(N18) showed a melting endothermic peak based on octadecyl side-chains (Figure 2). $T_{\mathrm{g}}$ of poly(N18) was 
Table I. Homopolymerization ${ }^{\mathrm{a}}$

\begin{tabular}{|c|c|c|c|c|c|c|c|c|}
\hline \multirow{2}{*}{ Monomer } & Time & Yield & $\bar{M}_{n}^{\mathrm{b}}$ & $\bar{M}_{w}{ }^{\mathrm{b}}$ & $T_{\mathrm{m}}$ & $T_{\mathrm{g}}$ & $\Delta H_{\mathrm{m}}$ & \multirow{2}{*}{$N^{\mathrm{c}}$ (lit.) } \\
\hline & $\mathrm{h}$ & $\%$ & $\times 10^{-4}$ & $\times 10^{-4}$ & ${ }^{\circ} \mathrm{C}$ & ${ }^{\circ} \mathrm{C}$ & $\mathrm{kcal} \mathrm{mol}^{-1}$ & \\
\hline $\mathrm{OA}$ & 5 & 48 & 12.9 & 6.1 & 51 & - & 4.83 & $6.5(9.4)^{11}$ \\
\hline OMA & 5 & 65 & 35.4 & 13.0 & 38 & - & 3.78 & $5.1(5.6)^{12}$ \\
\hline OAAm & 7 & 17 & 2.5 & 1.8 & 32 & - & 2.87 & $3.9(5.7)^{11}$ \\
\hline OMAm & 5 & 59 & 2.4 & 1.1 & 24 & - & 2.68 & 3.6 \\
\hline AAIT & 10 & 50 & 3.5 & 1.7 & - & 231 & & \\
\hline N18 & 5 & 68 & 5.9 & 3.5 & -15 & 132 & 1.44 & 2.0 \\
\hline
\end{tabular}

a Polymerization conditions: $[\mathrm{M}]_{0}=0.4 \mathrm{moll}^{-1},[\mathrm{AIBN}]=1.4 \mathrm{mmol}^{-1}$ in benzene for OA, OMA, OMAm, and $\mathrm{N} 18$, in DMAc for OAAm and AAIT at $60^{\circ} \mathrm{C}$.

b Determined by GPC.

c Number of crystallizing $\mathrm{CH}_{2}$ groups calculated from $\Delta H_{\mathrm{m}}$.

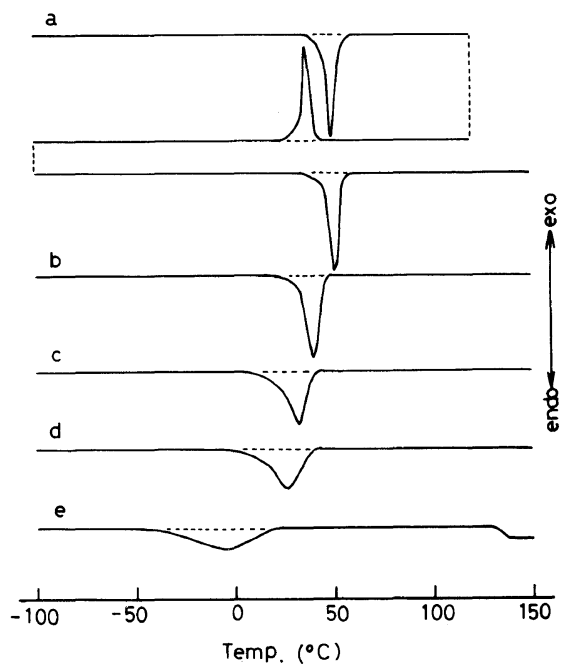

Figure 2. DSC curves of (a) poly(OA), (b) poly(OMA), (c) poly(OAAm), (d) poly(OMAm), and (e) poly(N18) at the scanning rate of $10^{\circ} \mathrm{C} \mathrm{min}-1$.

observed at $132^{\circ} \mathrm{C}$, whereas $T_{\mathrm{g}}$ could not be detected for other comb-like polymers whose glass transition would occur at $T_{\mathrm{m}}$ of the side-chains crystal as reported previously. ${ }^{1-4}$ Melting temperature $\left(T_{\mathrm{m}}\right)$ from the peak top and heat of fusion $\left(\Delta H_{\mathrm{m}}\right)$ estimated from the second heating run are shown in Table I. The side-chains of the converntional comb-like polymers such as poly(OA), poly(OMA), and poly(OAAm) have been found to be crystal- lized in hexagonal form. ${ }^{3}$ Crystal forms of the other polymers were confirmed by IR measurements. The IR spectra of poly(OMAm) and poly(N18) showed a single absorption peak at $720 \mathrm{~cm}^{-1}$, corresponding to the $\mathrm{C}-\mathrm{H}$ vibrations of the methylene chain, which indicate that the alkyl side-chains of the polymers crystallized in hexagonal form. ${ }^{3}$ Therefore, the number of crystallizing $\mathrm{CH}_{2}$ groups $(N)$ in the side-chains was calculated from $\Delta H_{\mathrm{m}}$ on the basis of the heat of fusion of $n$-alkanes in hexagonal packing $\left(735 \mathrm{cal} \mathrm{mol}^{-1} \mathrm{CH}_{2}{ }^{-1}\right){ }^{11}$ $N$ numbers are' also shown in Table I. The $T_{\mathrm{m}}$ and $N$ numbers were in the following order: $\operatorname{poly}(\mathrm{OA})>\operatorname{poly}(\mathrm{OMA})>\operatorname{poly}(\mathrm{OAAm})>$ poly(OMAm) $>\operatorname{poly}(\mathrm{N} 18)$. It has been reported that the crystallization of alkyl side-chains depends on the flexibility of the polymer main-chain, and the flexible mainchain of the comb-like polymers would be favorable to side-chain crystallization. ${ }^{11,13-15}$ It is well known that the introduction of $\alpha$ methyl group or amide group to the monomer units raises the $T_{\mathrm{g}}$ of polymers due to increase in rigidity. The order of crystallinity $\left(T_{\mathrm{m}}\right.$ and $N$ ) would agree with the order of the flexibility of the main-chain. In particular, poly(N18) showed low crystallinity because the side-chains crystallized below $T_{\mathrm{g}}$ of the main-chain. 
Table II. Monomer reactivity ratios for the copolymerization of isopropenyltriazines with monomers each having an octadecyl group

\begin{tabular}{clccc}
\hline $\mathrm{M}_{2}$ & $\mathrm{M}_{1}$ & Solvent & $r_{1}$ & $r_{2}$ \\
\hline \multirow{2}{*}{ AAIT } & OA & DMAc & $0.60 \pm 0.04$ & $1.77 \pm 0.25$ \\
& OMA & DMAc & $0.92 \pm 0.18$ & $0.62 \pm 0.13$ \\
& OAAm & DMAc & $0.43 \pm 0.11$ & $4.34 \pm 0.38$ \\
& OMAm & DMAc & $0.65 \pm 0.06$ & $3.79 \pm 0.37$ \\
$\mathrm{~N} 18$ & OA & Benzene & $0.06 \pm 0.03$ & $1.52 \pm 0.16$ \\
& OMA & Benzene & $0.29 \pm 0.07$ & $1.33 \pm 0.08$ \\
& OAAm & Benzone & $0.28 \pm 0.10$ & $3.16 \pm 0.78$ \\
& OMAm & Benzone & $0.31 \pm 0.10$ & $2.97 \pm 0.55$ \\
\hline
\end{tabular}

From the above, it seems that comb-like copolymer consisting of flexible unit and rigid unit is effective for obtaining polymers with higher $T_{\mathrm{g}}$ of main-chain and higher crystallinity of side-chains. Copolymerizations of AAIT $\left(\mathrm{M}_{2}\right)$ with OA, OMA, OAAm, and OMAm $\left(\mathrm{M}_{1}\right)$ were carried out in DMAc. The compositions of the copolymers were calculated from elemental analysis and monomer reactivity ratios $r_{1}$ and $r_{2}$ were determined by the integrated copolymerization equation of Mayo-Lewis. The reactivity ratios are shown in Table II. DSC curves in the second heating process for the comb-like copolymers are shown in Figure 3. $T_{\mathrm{g}}$ of main-chain could not be detected for poly(AAIT-co-OA) at lower AAIT content $\left(\mathrm{d} M_{2}=12\right.$ and $\left.27 \mathrm{~mol} \%\right)$ (Figure 3, a and b). Both melting of side-chains and $T_{\mathrm{g}}$ of main-chain were observed for the copolymers at AAIT content 47 and $57 \mathrm{~mol} \%$ (Figure 3, c and d). However, the side-chains could not crystallize at $66 \mathrm{~mol} \%$ AAIT content (Figure 3, e). Both $T_{\mathrm{m}}$ and $T_{\mathrm{g}}$ were also observed for the other comb-like copolymers in similar composition region as poly(AAITco-OA).

In Figure $4, T_{\mathrm{g}}, T_{\mathrm{m}}$, and $N$ number obtained from the DSC curves of the comb-like copolymers are plotted against the compositions of the copolymers. The IR spectra of the copolymers showed that the crystal form of the

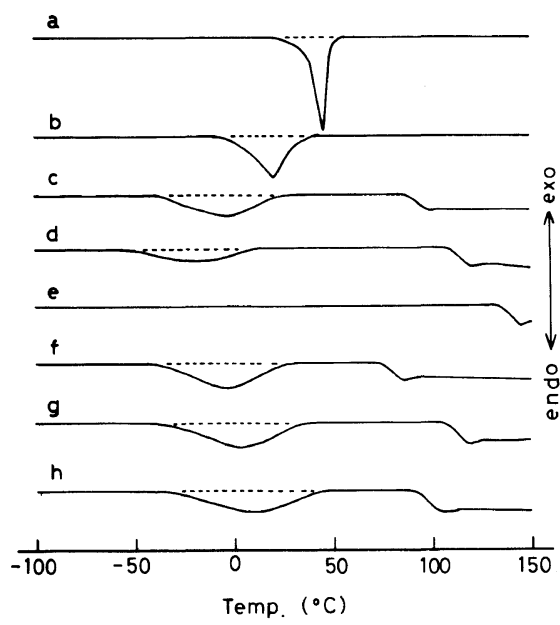

Figure 3. DSC curves for copolymers of AAIT $\left(\mathrm{M}_{2}\right)$ at a scanning rate of $10^{\circ} \mathrm{C} \mathrm{min}{ }^{-1}$. (a) AAIT-OA $\left(\mathrm{M}_{2}=\right.$ $12 \mathrm{~mol} \%)$, (b) AAIT OA $\left(\mathrm{M}_{2}=27 \mathrm{~mol} \%\right)$, (c) AAITOA $\left(\mathrm{M}_{2}=47 \mathrm{~mol} \%\right)$, (d) AAIT-OA $\left(\mathrm{M}_{2}=57 \mathrm{~mol} \%\right)$, (e) AAIT-OA $\left(\mathrm{M}_{2}=66 \mathrm{~mol} \%\right)$, (f) AAIT-OMA $\left(\mathrm{M}_{2}=43\right.$ mol\%), (g) AAIT-OAAm $\left(\mathrm{M}_{2}=35 \mathrm{~mol} \%\right)$, (h) AAITOMAm $\left(\mathrm{M}_{2}=16 \mathrm{~mol} \%\right)$.

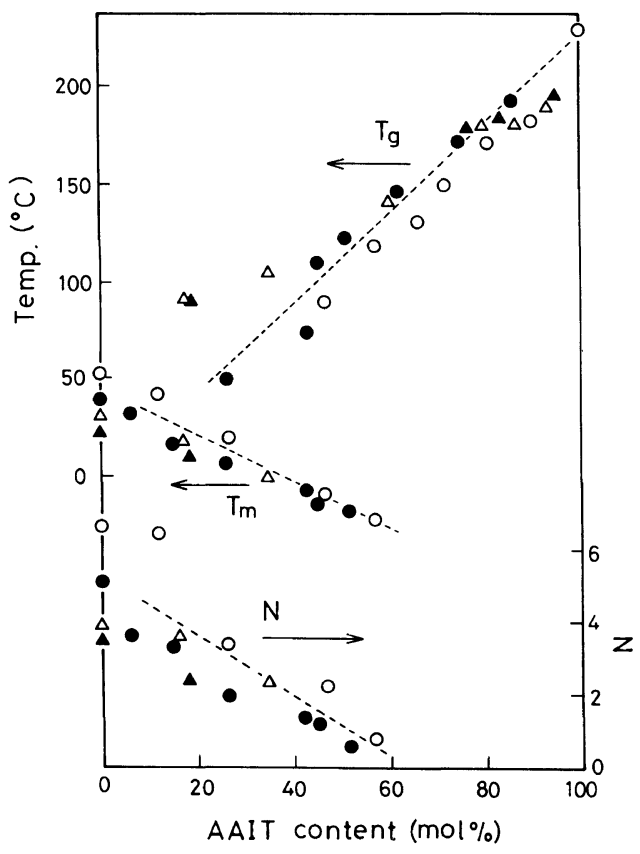

Figure 4. Relationship between glass transition temperature $\left(T_{\mathrm{g}}\right)$, melting temperature $\left(T_{\mathrm{m}}\right)$, and number of crystallizing $\mathrm{CH}_{2}$ groups $(\mathrm{N})$ and AAIT content in copolymer of AAIT with OA (O), OMA (O), OAAm $(\triangle)$, and $\operatorname{OMAm}(\boldsymbol{\Delta})$. 
side-chains was hexagonal packing similar to that of homopolymers. $T_{\mathrm{m}}$ of side-chains was observed in the region below $60 \mathrm{~mol} \%$ AAIT content, while $T_{\mathrm{g}}$ of main-chain was found above $20 \mathrm{~mol} \%$. That is, the side-chains of the comb-like copolymers could crystallize below $T_{\mathrm{g}}$ of the main-chain in the range of about 20 to $60 \mathrm{~mol} \%$ AAIT content. In this region, $T_{\mathrm{m}}$ was in -20 to $0^{\circ} \mathrm{C}$, and the $N$ number was from 0.5 to $2 . T_{\mathrm{g}}$ decreased with decreasing AAIT content, possibly due to the blocking effects of long alkyl chains on interactions of AAIT units such as hydrogen bonding of amino group with nitrogen atoms in the triazine ring. $T_{\mathrm{m}}$ and $N$ number increased with decreasing AAIT content. This indicates that the degree of crystallinity and perfection of crystals would be lowered by decrease in the concentrations of octadecyl side-chains.

Copolymers from isopropenyltriazine with comonomers, both of which have a octadecyl group, are expected to raise the crystallinity of side-chains. Accordingly, the copolymerizations of N18 with OA, OMA, OAAm, and OMAm were carried out in benzene. The monomer reactivity ratios for the copolymerizations are shown in Table II. The $r_{1}$ values of N18 are lower than those of AAIT. Previously, we reported that the $r_{1}$ values for the copolymerizations of 2-amino-4-( $N$-alkylanilino)-6-isopropenyl-1,3,5-triazines with methyl methacrylate (MMA) were lower than that of AAIT with MMA. ${ }^{6,9}$ In addition, when the solvent varied from DMAc to benzene, $r_{1}$ decreased for the copolymerization of 2-amino4-( $N$-alkylanilino)-6-isopropenyl-1,3,5-triazine with MMA. ${ }^{9}$ Therefore, decrease in $r_{1}$ for the present copolymerizations would be due to the effects of the $N$-alkyl substituent and solvent.

DSC curves of the resulting comb-like copolymers are shown in Figure 5. $T_{\mathrm{g}}$ of main-chain was observed above the melting endotherm for poly(N18-co-OA) at high N18 content (Figure 5, b, c, d, and e). The other comb-like copolymers showed a similar tendency to poly(N18-co-OA).

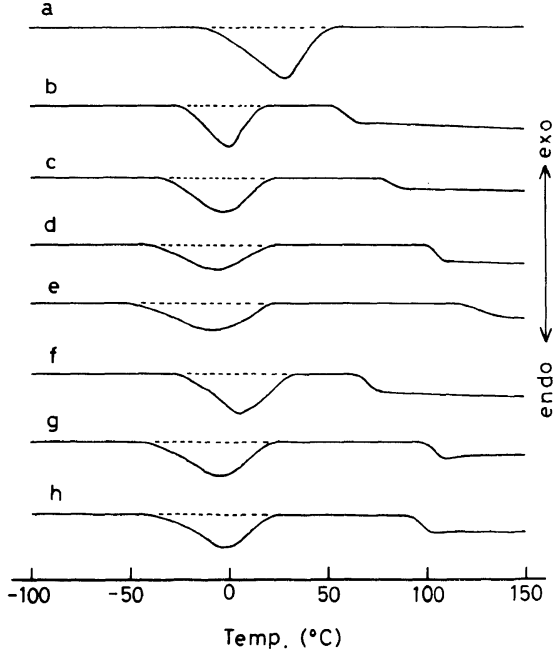

Figure 5. DSC curves for copolymers of N18 $\left(\mathrm{M}_{2}\right)$ at a scanning rate of $10^{\circ} \mathrm{C} \min ^{-1}$. (a) N18-OA $\left(\mathrm{M}_{2}=37\right.$ mol\%), (b) N18-OA $\left(\mathrm{M}_{2}=54 \mathrm{~mol} \%\right)$, (c) N18-OA $\left(\mathrm{M}_{2}=67 \mathrm{~mol} \%\right)$, (d) N18-OA $\left(\mathrm{M}_{2}=77 \mathrm{~mol} \%\right)$, (e) N18$\mathrm{OA}\left(\mathrm{M}_{2}=87 \mathrm{~mol} \%\right)$, (f) N18-OMA $\left(\mathrm{M}_{2}=46 \mathrm{~mol} \%\right)$, (g) N18-OAAm $\left(\mathrm{M}_{2}=62 \mathrm{~mol} \%\right)$, (h) N18-OMAm $\left(\mathrm{M}_{2}=\right.$ $60 \mathrm{~mol} \%$ ).

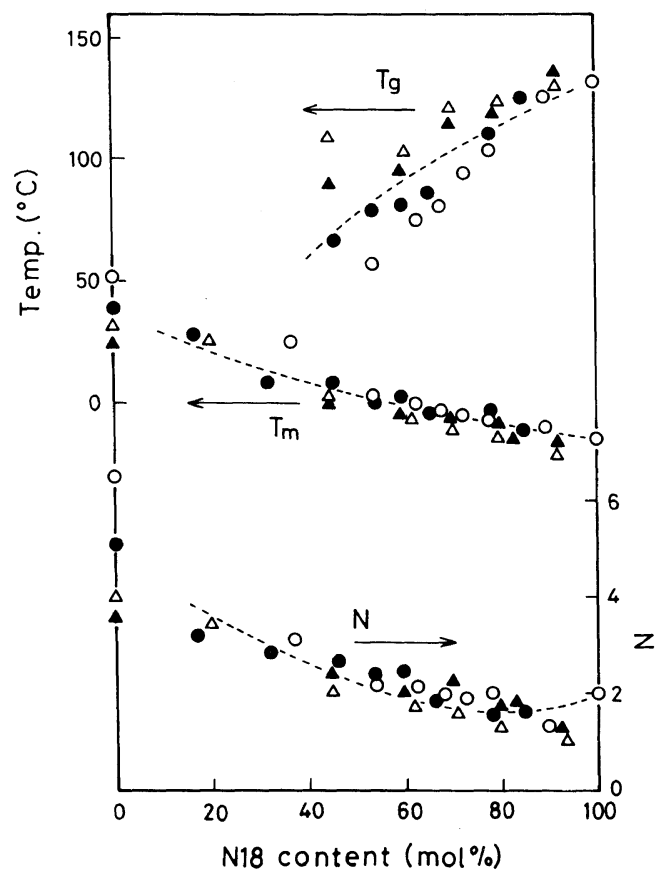

Figure 6. Relationship between glass transition temperature $\left(T_{\mathrm{g}}\right)$, melting temperature $\left(T_{\mathrm{m}}\right)$, and number of crystallizing $\mathrm{CH}_{2}$ groups $(N)$ and N18 content in copolymer of N18 with OA $(\bigcirc)$, OMA $(\bullet), \operatorname{OAAm}(\triangle)$, and OMAm (A). 
The relationships between $T_{\mathrm{g}}, T_{\mathrm{m}}$, and $N$ number and composition of the copolymer are illustrated in Figure 6. The side-chains of the copolymers could crystallize in the whole composition region. $T_{\mathrm{g}}$ was observed at N18 content more than $50 \mathrm{~mol} \%$ for all copolymers. $T_{\mathrm{m}}$ was from -20 to $10^{\circ} \mathrm{C}$ and $N$ number, from 1 to 3 in this content region. These values were slightly higher than those of copolymers obtained from AAIT. $T_{\mathrm{m}}$ and $N$ number decreased with increasing N18 content, although the copolymers consisted of monomer units, both of which have a long alkyl group. This would be due to difference in the flexibility of main-chain between N18 unit and longchain comonomer unit. The flexible main-chain of comb-like copolymers would be favorable to side-chain crystallization as mentioned in the crystallinity of homopolymers. Flexibility would be lowered by increase in N18 content, which is also supported by the rise in $T_{\mathrm{g}}$ of copolymers with N18 content. Consequently, the degree of crystallinity and perfection of crystals decrease with increasing N18 content. However, the $N$ numbers in the region of higher N18 content near the homopolymer is lower than that of poly(N18), as shown in Figure 6. The reason for this is not clear at the present stage. The flexibility of the main-chain might be lowered by the introduction of a small amount of comonomer unit which would act as a spacer favorable to intermolecular interactions of triazine units such as hydrogen bonding of amino group.

In summary, comb-like copolymers which have $T_{\mathrm{g}}$ of main-chain higher than $T_{\mathrm{m}}$ of side-chain crystals could be synthesized by the copolymerization of AAIT or N18 with comonomers having octadecyl groups such as OA, OMA, OAAm, and OMAm. The crystallinity of the side-chains of the resulting copolymers depended on the concentration of alkyl side-chain and flexibility of the polymer main-chain.

\section{REFERENCES}

1. H. S. Kaufman, A. Sasher, T. Alfrey, and J. Fankuchen, J. Am. Chem. Soc., 70, 3147 (1948).

2. E. F. Jordan, Jr., J. Polym. Sci., A-1, 9, 3367 (1971).

3. N. A. Platé and V. P. Shibaev, J. Polym. Sci., Macromol. Rev., 8, 117 (1974).

4. H. K. Reimschuessel, J. Polym. Sci., Polym. Chem. Ed., 17, 2447 (1979).

5. Y. Yuki, T. Kakurai, and T. Noguchi, Bull. Chem. Soc. Jpn., 43, 2123 (1970).

6. Y. Yuki, H. Kunisada, J. Taniuchi, and N. Takizuka, Nippon Kagaku Kaishi, 224 (1987).

7. H. Kunisada, Y. Yuki, and H. Arai, Nippon Kagaku Kaishi, 97 (1989).

8. H. Kunisada, Y. Yuki, S. Kondo, J. Miyatake, and C. Maeda, Polym. J., 22, 559 (1990).

9. H. Kunisada, Y. Yuki, S. Kondo, J. Miyatake, and C. Maeda, Polym. J., 22, 977 (1990).

10. E. F. Jordan, Jr., G. R. Riser, W. E. Parker, and A. N. Wrigley, J. Polym. Sci., A-2, 4, 975 (1966).

11. E. F. Jordan, Jr., D. W. Feldeisen, and A. N. Wrigley, J. Polym. Sci., A-1, 9, 1835 (1971).

12. K. Yokota, T. Kougo, and T. Hirabayashi, Polym. J., 15, 891 (1983).

13. E. F. Jordan, Jr., B. Arthymyshyn, A. Speca, and A. N. Wrigley, J. Polym. Sci., A-1, 9, 3349 (1971).

14. E. F. Jordan, Jr., G. R. Riser, B. Arthymyshyn, J. W. Pensabene, and A. N. Wrigley, J. Polym. Sci., A-2, 10, 1657 (1972).

15. T. Hirabayashi, T. Kikuta, K. Kasabou, and K. Yokota, Polym. J., 20, 693 (1988). 